\title{
CONTRIBUTION TO THE LICHEN FLORA OF NORTH EAST GREENLAND. V. ZAC- KENBERG AND CLAVERING Ø AREA
}

\section{Eric Steen Hansen}

Natural History Museum of Denmark, University of Copenhagen, Herbarium, Botanical Garden, Øster Farimagsgade 2 C, Copenhagen K DK-1123, Denmark

E-mail: erich@snm.ku.dk

\begin{abstract}
Hansen E.S., 2017: Contribution to the lichen flora of North East Greenland. V. Zackenberg and Clavering Ø area [Papildomi duomenys apie pietryčių Grenlandijos kerpių florą V. Zakenbergo ir Klaveringo Ø vietovės]. Bot. Lith., 23(1): 43-50.

The paper lists 202 lichen taxa from Zackenberg and Clavering $\varnothing$ area, North East Greenland. Ochrolechia androgyna, Psora globifera and Rhizocarpon renneri are new to East Greenland. Sixteen lichen taxa are new to North East Greenland, viz. Aspicilia aquatica, Candelariella dispersa, Chaenotheca furfuracea, Circinaria caesiocinerea, Cladonia libifera, Lecanora cenisia, L. chloroleprosa, L. leptacina, Lichenomphalia alpina, Miriquidica atrofulva, M. nigroleprosa, Ochrolechia alaskana, Peltigera castanea, P. extenuata, Phylliscum demangeonii and Pyrenopsis furfurea.
\end{abstract}

Keywords: Arctic region, diversity, lichens.

\section{INTRODUCTION}

In summer 1994, one year before the establishment of Zackenberg Research Station in high-arctic North East Greenland, the author visited the area for the following purposes: to extend the so-called ZERO-line to the top of Aucellabjerg (Meltofte et al., 2008), to establish permanent plots and stations on rocks and soil in the whole Zackenberg area for long-term monitoring of changes in climate-related effects on lichens, and, finally, to obtain a representative collection of lichens in Zackenberg area. The main purpose of the present paper is to provide a list of lichens collected by the author in July and August 1994. It aims to assist future visitors with a particular interest in the lichen flora of Zackenberg. The list includes a number of lichens collected by the author from Theodolit Plateau on Clavering Ø, just south of Zackenberg in late July 1994. It is not the intention to deal with the results of the monitoring work carried out in Zackenberg in 1994-2015. Interested readers are referred to the an- nual ZERO reports published by the National Environmental Research Institute, Aarhus University. The lichen flora of North East Greenland was previously investigated by B.Lynge and P.F.Scholander in 1929 and 1930 (Lynge \& Scholander, 1937; Lynge, 1940). V.Alstrup, F.Daniels and the author collected more than four hundred taxa of lichens in Kronprins Christian Land and Lambert Land north of Zackenberg in summer 1995 (Alstrup et al., 2000). An additional material on lichens from North East Greenland has been collected by C.Bay, R.Corner, B.Fredskild, A.Lundager. All these collections, except that of A.Lundager (reported by GALLøE (1910)), have been published by the author (HANSEN, 2008, 2009a, 2012).

Collecting of lichens was carried out by the author in two localities, Zackenberg $\left(74^{\circ} 30^{\prime} \mathrm{N}, 20^{\circ} 30^{\prime} \mathrm{W}\right.$; loc. No. 1 in list of species) - 20 July - 23 August 1994 and Theodolit Plateau $\left(74^{\circ} 24^{\prime} \mathrm{N}, 21^{\circ} 42^{\prime} \mathrm{W}\right.$; loc. No. 2 in list of species) - 29 July 1994. Lichens were studied in the lowland and up to $1040 \mathrm{~m}$ a. s. 1. in Zackenberg area, which is divided into two geologically 
different parts by a fault zone: the area with Cretaceous and Tertiary sandstones to the east, and the area dominated by Caledonian gneissic and granite bedrock to the west (КосH \& Haller, 1971). The sandstones are topped by Tertiary basalts. Glacial siliceous erratics up to boulder size occur commonly in a $2-3 \mathrm{~km}$ wide valley, where Zackenberg Station is situated today. This lowland was deglaciated about 10000 years ago. The rocks of Theodolit Plateau are composed of Archaen gneisses and mica schists, but rocks from the Carbon and the Cretaceous periods also occur in the area. Climatically and floristically, the investigation area is located in the high arctic, continental region (Meltofte et al., 2008). The mean annual temperature is $-10.3^{\circ} \mathrm{C}$ according to meteorological observations from Daneborg situated about $20 \mathrm{~km}$ from Zackenberg. The mean temperature in the warmest month July is $4^{\circ} \mathrm{C}$, while the mean temperature in the coldest month February is $-23.6^{\circ} \mathrm{C}$. The mean annual amount of precipitation is $214 \mathrm{~mm}$, most of it falling as snow in autumn and winter (JAKOBSEN, 1992). Winds from north and north-northeast dominate and result in heavy snowdrifting during winter. The layer of permafrost is up to $70 \mathrm{~cm}$ thick (Christiansen \& Humlum, 1993). Earlier melting of ice and snow resulting in a longer growing season has been one of many consequences of global warming during recent years.

Mixed dwarf shrub heaths more or less rich in lichens are widely distributed in Zackenberg area. Thus, well-developed Dryas-Carex rupestris heaths rich in lichens with a distinct preference for soil having a neutral or slightly alkaline reaction, for example, Caloplaca ammiospila, Hypogymnia subobscura, Megaspora verrucosa, Ochrolechia upsaliensis, Phaeorrhiza nimbosa, Physconia muscigena and Rinodina mniaraea, occur commonly on the flat lowland plain. It is a comparatively dry type of heath, which is often exposed to heavy winds and covered by a thin layer of snow during winter. Contrary to the mixed Dryas heaths, the Cassiope-Salix arctica heaths, which are widely distributed at a slightly higher level in the lowland below Aucellabjerg, are characterized by a somewhat thicker snow cover during winter. These heaths contain lichens such as Cetrariella delisei, Cladonia amaurocraea, C. mitis, C. trassii, Ochrolechia alaskana, Psoroma tenue var. boreale and Sphaerophorus fragilis. Fell field patches with Bryoria chalybeiformis, Candelariella canadensis, Cetraria muricata,
Stereocaulon arenarium and Thamnolia vermicularis var. subuliformis occur both in the lowland and at higher levels on Aucellabjerg. Steppe-like vegetation dominated by sedges such as Kobresia myosuroides and Carex rupestris includes lichens with a distinct preference for neutral to alkaline soil such as Caloplaca erichansenii, Catapyrenium cinereum, Fulgensia bracteata, Placidium lachneum, Psora decipiens, P. rubiformis and Toninia sedifolia. This plant community is rich in soil crust lichens previously discussed by the author (HANSEN, 2001). In Zackenberg area, it is restricted to south and southeast exposed slopes. A particularly well-developed soil crust community with Psora vallesiaca, Phaeorriza nimbosa and Toninia sedifolia was found on Theodolit Plateau. Open places in fens were covered by different Solorinas, for example, Solorina saccata and S. spongiosa, and Peltigeras, for example, Peltigera aphthosa, P. britannica and $P$. leucophlebia. Snow patches with Cetrariella delisei, Cladonia borealis and Solorina crocea occurred on more or less moist, north-facing and sloping ground, often in association with Cassiope tetragona.

Saxicolous lichens occurred in the whole investigated area from sea level to the top of the mountains. The numerous siliceous stones and boulders supported species such as Brodoa oroarctica, Lecidea tesselata, Pseudephebe minuscula, Rhizocarpon geographicum. R. jemtlandicum, Umbilicaria hyperborea, $U$. lyngei and U. proboscidea. Aspicilia aquatica and Staurothele areolata were found growing on a temporarily moist boulder on Zackenberg Elv. Boulders manured by birds, arctic foxes and musk oxen held a nitrophilous lichen flora consisting of species such as Acarospora peliscypha, Dimelaena oreina, Lecidea atrobrunnea, Melanohalea elegantula, Montanelia disjuncta, Physcia caesia, Protoparmelia badia, Rhizoplaca melanophthalma, Sporastatia testudinea, Umbilicaria krascheninnikovii, Xanthoria candelaria and $X$. elegans. Ferrugineous lichens, for example, Miriquidica atrofulva, Porpidia flavocaerulescens, P. melinodes and Tremolecia atrata, occurred more or less scattered in the investigation area. A number of lichens grew on both siliceous and basaltic rocks, for example, Umbilicaria virginis and Usnea sphacelata. The last mentioned species grows abundantly on the top of Aucellabjerg (Hansen, 1996). A number of lichens were restricted to basaltic rocks, for example, Aspicilia candida, A. pergibbosa, Bellemerea sub- 
sorediza, Xanthoria sorediata and X. subfruticulosa. Some lichens were found growing on old musk ox bones, which occurred scattered on the ground, for example, Caloplaca alcarum, C. cerina, C. tiroliensis, C. fraudans, Lecanora hagenii var. fallax, Physcia dubia and Xanthoria candelaria. Generally, the lichen flora reflects the continental climatic conditions that characterize this part of North East Greenland.

A total of 400 lichen specimens from Zackenberg area and 120 lichen specimens from Theodolit Plateau on Clavering $\varnothing$ were studied using Zeiss light microscopes, and identified by the author. The nomenclature in the list is presented after NoRDIN et al. (2011) with some exceptions such as the listed species of Caloplaca. The author prefers to use this name instead of the new genus names published by ARUP et al. (2013) in order to facilitate a comparison with other papers dealing with this group of lichens in North East Greenland. The specimens are deposited at the Botanical Museum of the University of Copenhagen (C).

Lichens new to North East Greenland are marked with one asterisk $(*)$. Two asterisks $(* *)$ indicate taxa new to East Greenland; "ap." and "pe." mean presence of apothecia and perithecia, respectively; "st." means that the specimen is sterile. Annotations are given regarding substrate of the lichens. The frequency in the investigation area is given for selected lichens of particular interest just as information about the distribution in Greenland is given for selected lichens. Numbers 1 and 2 refer to the collection localities (see above). Collections distributed from the Herbarium $\mathrm{C}$ as part of "Lichenes Groenlandici Exsiccati" (LGE) are stated by their numbers.

\section{List of species}

Acarospora peliscypha Th.Fr. - on manured siliceous rock; 1; ap.

Acarospora rhizobola (Nyl.) Alstrup - on soil; 1; ap.

Amandinea cacuminum (Th.Fr.) H.Mayrhofer \& Sheard - on manured siliceous rock; 2; ap.

Amandinea punctata (Hoffm.) Coppins \& Scheid. - on musk ox dung and plant remains; 1; ap.

Arctocetraria nigricascens (Nyl.) Kärnefelt \& Thell - on soil; 1; st.; scattered.

Arthrorhaphis alpina (Schaer.) R.Sant. - on mineral soil; 1; st.; common. LGE 844.

Arthrorhaphis citrinella (Ach.) Poelt - on soil together with Candelariella terrigena and Lecanora geophila; st.

* Aspicilia aquatica Körb. - on moist siliceous rock together with Staurothele areolata; 1; ap.

Aspicilia candida (Anzi) Hue - on basaltic rock; 1; ap.

Aspicilia pergibbosa (H.Magn.) Räsänen - on basaltic rocks; 1; ap.

Baeomyces carneus Flörke - on mineral soil and soil rich in humus; 1,2 ; st.; scattered.

Baemyces placophyllus Ach. - on clayey soil; 1; st.; scattered.

Bellemerea subsorediza (Lynge) R.Sant. - on basaltic rock; 1 ; st.

Biatora vernalis (L.) Fr. - on soil; 1; ap.; scattered.

Brodoa oroarctica (Krog) Goward - on siliceous rock; 1; ap.; scattered.

Bryonora castanea (Hepp) Poelt - on plant remains and mosses; 2; ap.

Bryoriachalybeiformis (L.) Brodo \& D.Hawksw.on soil; 1, 2; st.; common.

Buellia leptocline (Flot.) Körb. - on moist siliceous rock; 1; ap.

Buellia papillata (Sommerf.) Tuck. - on plant remains and mosses; 1, 2; ap.; common.

Caloplaca alcarum Poelt - on old musk ox bones; $1 ;$ ap.

Caloplaca ammiospila (Wahlenb.) H.Olivier - on plant remains; 1; ap.

Caloplaca castellana (Räsänen) Poelt - on Placynthium asperellum and Rhizocarpon geminatum on siliceous and basaltic rocks; 1; ap.

Caloplaca cerina (Ehrh. ex Hedw.) Th.Fr. - on plant remains, mosses, hare droppings, musk ox dung, wood and old bones; 1, 2; ap.; scattered.

Caloplaca epithallina Lynge - on Rhizoplaca melanophthalma on siliceous rock; 1; ap.

Caloplaca erichansenii S.Y.Kondr., A.Thell, Kärnefelt \& Elix - on soil, plant remains and mosses; 1,2 ; st.

Caloplaca fraudans (Th.Fr.) H.Olivier - on old bone; 1; ap.

Caloplaca jungermanniae (Vahl) Th.Fr. - on plant remains and mosses; 2; ap.

Caloplaca psoricida E.S.Hansen, Poelt \& Søchting - on Psora rubiformis on mineral soil; 1, 2; st.

Caloplaca tetraspora (Nyl.) H.Olivier - on plant remains; 1, 2; ap. 
Caloplaca tiroliensis Zahlbr. - on plant remains, hare droppings, musk ox dung and old bones; 1, 2; ap.; scattered.

Candelariella aurella (Hoffm.) Zahlbr. - on old musk ox bones; 1 ; ap.

Candelariella borealis M.Westb. - on soil; 1; st. LGE 559.

Candelariella canadensis H.Magn. - on soil, plant remains and mosses; 1, 2; ap.

* Candelariella dispersa (Räsänen) Hakul. - on Placynthium asperellum on siliceous rocks; 1, 2; ap.; common. LGE 559.

Candelariella vitellina (Hoffm.) Müll.Arg. - on manured siliceous and basaltic rocks; 1, 2; ap.

Candelariella xanthostigma (Ach.) Lettau - on musk ox dung and wood; 1, 2; st.

Catapyrenium cinereum (Pers.) Körb. - on clayey soil; 1; ap., scattered.

Catapyrenium daedaleum (Kremp.) Stein - on mineral soil together with Phaeorrhiza nimbosa; 1, 2; pe.

Cetraria islandica (L.) Ach. - on soil; 1, 2; st.; common.

Cetraria muricata (Ach.) Eckfeldt - on soi; 1, 2; st.; common.

Cetrariella delisei (Bory ex Schaer.) Kärnefelt \& Thell - on soil; 1, 2; st.; common. LGE 567.

* Chaenotheca furfuracea (L.) Tibell - on plant remains; 1 ; st. - C. furfuracea is often found growing on peat in tombs and abandoned peat-cottages (HANsen, 1982).

* Circinaria caesiocinerea (Nyl. ex Malbr.) A.Nordin, S.Savić \& Tibell - on manured siliceous and basaltic rocks; 1, 2; ap.

Cladonia amaurocraea (Flörke) Schaer. - between mosses on soil; 1, 2; st.; scattered.

Cladonia borealis S. Stenroos - on soil; 1, 2; st.; common.

Cladonia carneola (Fr.) Fr. - on soil rich in humus; 1 ; st.

Cladonia chlorophaea (Flörke ex Sommerf.) Spreng. - between mosses on soil; 1, 2; st.; common.

Cladonia cornuta (L.) Hoffm. - on soil rich in humus; 1; st.

Cladonia cyanipes (Sommerf.) Nyl. - on soil rich in humus; 1 ; st.

Cladonia fimbriata (L.) Fr. - on soil rich in humus; 1 ; st.
* Cladonia libifera Savicz - on soil; 2; st. - C. libifera is known from a few localities in Greenland, for example, Storfjord Radio in South East Greenland and Constable Bugt in North Greenland (HANSEN, 2009b; HANSEN \& Ahti, 2011).

Cladonia luteoalba Wheldon \& A.Wilson - on soil rich in humus; 1 ; st.

Cladonia macroceras (Delise) Hav. - between mosses on soil; 1 ; st.

Cladonia macrophyllodes Nyl. - on soil; 1; st.

Cladonia mitis Sandst. - on soil; 1, 2; st.; scattered. LGE 568.

Cladonia pocillum (Ach.) O.J.Rich. - on plant remains; 1,2 ; st.; very common.

Cladonia pyxidata (L.) Hoffm. - on soil; 1, 2; st.; very common.

Cladonia scotteri Ahti \& E.S.Hansen - on soil; 1; ap.; very rare. $-C$. scotteri is fairly common in West Greenland, but rare and probably overlooked in East Greenland (Hansen \& Aнti, 2011).

Cladonia trassii Ahti - between mosses on soil; 1, 2; st.; common.

Cladonia uncialis (L.) F.H.Wigg. - on soil; 1; st.

Collema undulatum Laurer ex Flot. var. granulosum Degel. - on soil; 1; st.

Dimelaena oreina (Ach.) Norman - on manured siliceous rocks; 1, 2; ap.; scattered.

Diploschistes muscorum (Scop.) R.Sant. - on soil and mosses; 1; ap.; scattered. LGE 573.

Epilichen scabrosus (Ach.) Clem. - on Baeomyces carneus on clayey soil; 1 ; ap.

Euopsis pulvinata (Schaer.) Vain. - on siliceous rock; 1 ; st.

Flavocetraria cucullata (Bellardi) Kärnefelt \& Thell - on soil and plant remains; 1, 2; st., very common. LGE 572.

Flavocetraria nivalis (L.) Kärnefelt \& Thell - on soil and mosses; 1, 2; ap.; common.

Fulgensia bracteata (Hoffm.) Räsänen - on mineral soil; 1; ap.; scattered.

Fuscopannaria praetermissa (Nyl.) P.M.Jørg. on soil; 1,2 ; ap.

Gowardia nigricans (Ach.) P.Halonen, L.Myllus, S.Velmela \& H.Hyvärinen - on soil; 1; st.; scattered.

Hypogymnia austerodes (Nyl.) Räsänen - on soil; 1,2 ; st.

Hypogymnia subobscura (Vain.) Poelt - on soil, gravel and plant remains; 1; st.; scattered. 
Ionaspis lacustris (With.) Lutzoni - on siliceous rock; 1 ; ap.

Lecanora argopholis (Ach.) Ach. - on siliceous rock and on Placynthium asperellum on basaltic rock; 1 ; ap.

* Lecanora cenisia Ach. - between mosses on siliceous rock; 2; ap.

* Lecanora chloroleprosa (Vain,) H.Magn. - on moist siliceous rock; 2 ; st.

Lecanora contractula Nyl. - on siliceous rocks and old musk ox bones; 1, 2; ap.

Lecanora epibryon (Ach.) Ach. - on soil, mosses and plant remains; 1, 2; ap.; very common.

Lecanora geophila (Th.Fr.) Poelt - on soil and plant remains; 1, 2; st.; scattered. LGE 571.

Lecanora hagenii (Ach.) Ach. var. fallax Hepp on dead mosses and old bones; 1, 2; ap.

Lecanora intricata (Ach.) Ach. - on siliceous and basaltic rocks; 1 ; ap.

Lecanora leptacina Sommerf. - between mosses on moist siliceous rock; 1; ap.

Lecanora leucococca Sommerf. - on siliceous and basaltic rocks; 1 ; ap.

Lecanora luteovernalis Brodo - on soil; 1; ap.

Lecanora marginata (Schaer.) Hertel \& Rambold - on siliceous and basaltic rocks; 1; ap.

Lecanora polytropa (Ehrh. ex Hoffm.) Rabenh. on siliceous and basaltic rocks; 1, 2; ap.; common.

Lecidea atrobrunnea (Ramond ex Lam. \& DC.) Schaer. - on manured siliceous and basaltic rocks; 1 , 2; ap.; scattered.

Lecidea auriculata Th.Fr. - on siliceous rock; 2; ap.

Lecidea lapicida (Ach.) Ach. var. lapicida - on siliceous rock; 1 ; ap.

Lecidea tessellata Flörke - on siliceous and basaltic rocks; 1, 2; ap.

Lecidella bullata Körb. - on siliceous and basaltic rocks; 1 ; ap.

Lecidella euphorea (Flörke) Hertel - on old musk ox bone; 1; ap.

Lecidella stigmatea (Ach.) Hertel \& Leuckert on siliceous rock; 2 ; st.

Lecidella wulfenii (Hepp) Körb. - on plant remains; 1 ; ap.

Lecidoma demissum (Rutstr.) Gotth. Schneid. \& Hertel - on soil; 1, 2; ap.

Leciophysma finmarkicum Th.Fr. - on soil; 1; st.
Lepraria alpina (de Lesd.) Tretiach \& Baruffo var. zeorinica L.Saag - on clayey soil; 1 .

Lepraria eburnea J.R.Laundon - on mineral soil; 1 ; common.

Lepraria gelida Tønsberg \& Zhurbenko - on mosses; $1,2$.

Lepraria neglecta (Nyl.) Erichsen - on soil; 1.

Lepraria vouauxii (Hue) R.C.Harris - on soil; 1, 2.

Leprocaulon gracilescens (Nyl.) I.M.Lamb \& A.M.Ward - on clayey soil; 2. - L. gracilescens has previously been reported from Lakseelvdalen in Southwest Greenland and Theodolit Plateau (SAAG et al., 2007).

Leprocaulon subalbicans (I.M.Lamb) I.M.Lamb \& A.M.Ward - on mosses; 1, 2.

* Lichenomphalia alpina (Britzelm.) Redhead, Lutzoni, Moncalvo \& Vilgalys - on soil; 1 .

Megaspora verrucosa (Ach.) Hafellner \& V.Wirth - on soil; 1; ap.; scattered.

Melanelia hepatizon (Ach.) A.Thell - on siliceous rocks; 1,2 ; st.

Melanohalea elegantula (Zahlbr.) O.Blanco, A.Crespo, Divakar, Essl., D.Hawksw. \& Lumbsch on manured siliceous rocks; 1,2 ; st.

* Miriquidica atrofulva (Sommerf.) A.J.Schwab $\&$ Rambold - on siliceous rock rich in iron together with Tremolecia atrata; 2 ; st.

* Miriquidica nigroleprosa (Vain.) Hertel \& Rambold var. nigroleprosa - on siliceous rock; 2; st.

Montanelia disjuncta (Erichsen) Divakar, A.Crespo, Wedin \& Essl. - on siliceous rocks; 1, 2; st.

Myxobilimbia lobulata (Sommerf.) Hafellner. on mineral soil; 1 ; ap.

Nephroma expallidum (Nyl.) Nyl. - on soil; 1; st.; rare.

* Ochrolechia alaskana (Verseghy) Kukwa - on plant remains and mosses; 1, 2; st.; very common.

** Ochrolechia androgyna (Hoffm.) Arnold - on mosses and soil; 1,2 ; st. $-O$. androgyna is widely distributed in West Greenland, but has apparently been overlooked in East Greenland (Thомson, 1997).

Ochrolechia inaequatula (Nyl.) Zahlbr. - between mosses on soil; 2; st.

Ochrolechia upsaliensis (L.) A.Massal - on plant remains; 1; ap.; scattered.

Ophioparma ventosa (L.) Norman - on siliceous rocks; 1, 2; ap. 
Orphniospora moriopsis (A.Massal.) D.Hawksw.on siliceous rock; 2; ap.

Parmelia saxatilis (L.) Ach. - on siliceous rock; 1; st.; very rare.

Parmeliella triptophylla (Ach.) Müll.Arg. - on plant remains; 1 ; st.

Peltigera aphthosa (L.) Willd. - on soil and mosses; 1 ; st.

Peltigera britannica (Gyeln.) Holt. - Hartw. \& Tønsberg - between mosses on soil; 1; st.

* Peltigera castanea Goward, Goffinet \& Miadl.on soil on dead mosses; 1,2 ; st.

* Peltigera extenuata (Vain.) Lojka - between mosses on soil; 1,2 ; st.; scattered.

Peltigera lepidophora (Nyl. ex Vain.) Bitter - on mineral soil; 1,2 ; st.; scattered.

Peltigera leucophlebia (Nyl.) Gyeln. - on soil; 1, 2; st.; common.

Peltigera malacea (Ach.) Funck - between mosses on soil; 1,2 ; st.

Peltigera rufescens (Weiss) Humb. - on soil and mosses; 1; st.; common. LGE 566.

Peltigera venosa (L.) Baumg. - on soil; 1; ap.

Pertusaria coriacea (Th.Fr.) Th.Fr. - on soil; 1; ap.

Pertusaria dactylina (Ach.) Nyl. - on soil; 1; st.

Pertusaria geminipara (Th.Fr.) C.Knight ex Brodo - on soil and mosses; 1, 2; st.

Pertusaria oculata (Dicks.) Th.Fr. - on soil; 1; st.

Phaeophyscia sciastra (Ach.) Moberg - on manured siliceous and basaltic rocks, soil and plant remains; 1, 2; st.

Phaeorrhiza nimbosa (Fr.) H.Mayrhofer \& Poelt - on soil and mosses; 1, 2; ap.

Phaeorrhiza sareptana (Tomin) H.Mayrhofer \& Poelt var. sphaerocarpa (Th.Fr.) H.Mayrhofer \& Poelt - on mineral soil; 1; ap.

* Phylliscum demangeonii (Moug. \& Mont.) Nyl. - on gneissic rock; 2; ap.

Physcia caesia (Hoffm.) Fürnr. - on manured siliceous rock; 1 ; st.

Physcia dubia (Hoffm.) Lettau - on manured siliceous and basaltic rocks; also on soil, plant remains, mosses, musk ox dung, pellet and old bone; 1, 2; st.

Physconia muscigena (Ach.) Poelt - on soil, plant remains and mosses; 1, 2; st.; scattered.

Placidiopsis pseudocinerea O.Breuss - on mineral soil; 1 ; pe.
Placidium lachneum (Ach.) de Lesd. - on mineral soil; 1 ; pe.

Placynthium asperellum (Ach.) Trevis.- on siliceous and basaltic rocks; 1 ; ap.

Placynthium pannariellum (Nyl.) H.Magn. - on basaltic rock; 1 ; ap.

Pleopsidium chlorophanum (Wahlenb.) Zopf on siliceous rocks; 1, 2; ap.; rare.

Polychidium muscicola (Sw.) Gray - on soil and plant remains; 1, 2; ap.

Porpidia flavocaerulescens (Hornem.) Hertel \& A.J.Schwab - on siliceous rock rich in iron; 2 ; st.

Porpidia melinodes (Körb.) Gowan \& Ahti - on basaltic rock; 1 ; st.

Protoblastenia terricola (Anzi) Lynge - on clayey soil; 1 ; ap.

Protopannaria pezizoides (Weber) P.M. Jørg. \& S.Ekman - on soil and plant remains; 1, 2; ap.

Protoparmelia badia (Hoffm.) Hafellner - on manured siliceous rocks; 1, 2; ap.

Pseudephebe minuscula (Nyl. ex Arnold) Brodo \& D.Hawksw. - on siliceous and basaltic rocks; 1, 2; st.; common.

Pseudephebe pubescens (L.) M.Choisy - on siliceous rock; 2; st.

Psora decipiens (Hedw.) Hoffm. - on mineral soil; 1; ap.; scattered.

** Psora globifera (Ach.) A.Massal. - on soil; 2 ; ap. $-P$. globifera has a scattered occurrence in Southwest Greenland, but has probably been neglected in East Greenland (Hansen, 1995).

Psora rubiformis (Ach.) Hook. - on clayey soil; 1, 2; st.; scattered. LGE558.

Psora vallesiaca (Schaer.) Timdal - on mineral soil together with Phaeorrhiza nimbosa and Toninia sedifolia; 2; ap.

Psoroma tenue Henssen var. boreale Henssen on soil and plant remains; 1, 2; ap.

* Pyrenopsis furfurea (Nyl.) Leight. - on soil; 1; st.

Rhizocarpon geminatum Körb. - on manured siliceous and basaltic rocks; 1; ap.

Rhizocarpon geographicum (L.) DC. - on siliceous and basaltic rocks; 1, 2; ap.; common.

Rhizocarpon grande (Flörke) Arnold - on siliceous rock; 1 ; ap.

Rhizocarpon inarense (Vain.) Vain. - on siliceous rock; 2; ap. 
Rhizocarpon jemtlandicum (Malme) Malme - on siliceous rocks; 1, 2; ap.

Rhizocarpon lavatum (Fr.) Hazsl. - on moist siliceous rock; 1; ap.

Rhizocarpon pusillum Runemark - on Sporastatia testudinea on siliceous and basaltic rocks; 1; ap.

** Rhizocarpon renneri Poelt - on Dimelaena oreina on siliceous rock; 1 ; ap. $-R$. renneri has previously been reported from a few localities in northern parts of Greenland, for example, Qaanaaq and Constable Bugt (Hansen, 1989, 2009b).

Rhizocarpon superficiale (Schaer.) Vain. - on siliceous rock; 1; ap.

Rhizoplaca melanophthalma (DC.) Leuckert \& Poelt - on manured siliceous and basaltic rocks; 1 , 2; ap.; common.

Rinodina archaea (Ach.) Arnold - on plant remains; 1; ap.

Rinodina mniaraea (Ach.) Körb. - on mineral soil and plant remains; 1, 2; ap.

Rinodina turfacea (Wahlenb.) Körb. - on soil and plant remains; 1, 2; ap.; scattered.

Solorina bispora Nyl. - on clayey soil; 1, 2; ap.; scattered.

Solorina crocea (L.) Ach. - on mineral soil; 1, 2; ap.

Solorina saccata (L.) Ach. - between mosses on soil; 1; ap.

Solorina spongiosa (Ach.) Anzi - on alkaline soil; 1; ap.

Sphaerophorus fragilis (L.) Pers. - between mosses on soil; 1, 2; st.; scattered.

Sporastatia testudinea (Ach.) A.Massal. - on siliceous and basaltic rocks; 1,2 ; ap.

Staurothele areolata (Ach.) Lettau - on moist siliceous rock; 1; pe.

Staurothele fuscocuprea (Nyl.) Zschacke - on basaltic rock; 1; pe.

Stereocaulon alpinum Laurer - on soil; 1, 2; st.; very common.

Stereocaulon arenarium (Savicz) I.M.Lamb - on mineral soil; 1 ; st.

Stereocaulon botryosum Ach. - on siliceous rock; $1 ;$ st.

Stereocaulon condensatum Hoffm. - on gravelly soil; 1 ; ap.
Stereocaulon glareosum (Savicz) H.Magn. - on mineral soil and gravel; 1, 2; ap.

Stereocaulon vesuvianum Pers. - on siliceous rock rich in iron; 2 ; st.

Thamnolia vermicularis (Sw.) Schaer. var. subuliformis (Ehrh.) Schaer. - on soil; 1; very common.

Toninia sedifolia (Scop.) Timdal - on mineral soil; 1, 2; ap.

Tremolecia atrata (Ach.) Hertel - on moist siliceous rocks; 1, 2; ap.

Umbilicaria decussata (Vill.) Zahlbr. - on manured siliceous and basaltic rocks; 1, 2; ap.

Umbilicaria deusta (L.) Baumg. - on moist siliceous rock; 2 ; st.

Umbilicaria hyperborea (Ach.) Hoffm. - on siliceous rocks; 1, 2; ap.

Umbilicaria krascheninnikovii ( Savicz) Zahlbr. (syn. U. polaris (Schol.) Zahlbr.) - on manured siliceous rocks; 1, 2; ap.

Umbilicaria lyngei Schol. - on siliceous rock; 1; st.

Umbilicaria proboscidea (L.) Schrad. - on siliceous rocks; 1, 2; ap.

Umbilicaria torrefacta (Lightf.) Schrad. - on siliceous rocks; 1, 2; ap.

Umbilicaria virginis Schaer. - on siliceous and basaltic rocks; 1, 2; ap.; common.

Usnea sphacelata R. Br. - on siliceous and basaltic rocks; 1 ; st.

Xanthoria candelaria (L.) Fr. - on manured siliceous rock, plant remains, pellet, musk ox dung and old musk ox bones; 1, 2; st.; common.

Xanthoria elegans (Link) Th.Fr. - on manured siliceous and basaltic rocks and plant remains; 1, 2; ap.; very common. LGE 561.

Xanthoria sorediata (Vain.) Poelt - on basaltic rocks; 1 ; st.

Xanthoria subfruticulosa (Elenkin) Piin - on basaltic rocks; 1 ; st.; scattered.

\section{ACKNOWLEDGEMENTS}

I wish to thank Teuvo Ahti, Othmar Breuss, Theodore Esslinger, Per Magnus Jørgensen, Walter Obermayer, Lauri Saag, Tor Tønsberg and Orvo Vitikainen for their help to determine or verify the selected lichens, and Peer Corfixen for technical assistance. 


\section{REFERENCES}

Alstrup V., Hansen E.S., Daniels F.J.A., 2000: Lichenized, lichenicolous and other fungi from North and North-East Greenland. - Folia Cryptogamica Estonica, 37: 1-20.

Arup U., Søchting U., FrödÉN P., 2013: A new taxonomy of the family Teloschistaceae. - Nordic Journal of Botany, 31: 16-83.

Christiansen H.H., Humlum O., 1993: Glacial history and periglacial landforms of the Zackenberg area, Northeast Greenland: Preliminary results. Geografisk Tidsskrift, 93: 19-29.

Galløe O., 1910: Lichens from North-East Greenland (N. of $76^{\circ} \mathrm{N}$. lat.) collected by the DenmarkExpedition 1906-1908. - Meddelelser om Grønland, 43: 181-191.

HANSEN E.S., 1982: Lichens from Central East Greenland. - Meddelelser om Grønland. - Bioscience, 9: 1-33.

HANSEN E.S., 1989: The lichen flora of Qaanaaq (Thule), northwestern Greenland. - Mycotaxon, 35(2): 379-394.

HANSEN E.S., 1995: Greenland Lichens. - Copenhagen. HANSEN E.S., 1996: Vertical Distribution of Lichens on the Mountain, Aucellabjerg, Northeastern Greenland. - Arctic and Alpine Research, 28(1): 111-117.

HANSEN E.S., 2001: Lichen-Rich Soil Crusts of Arctic Greenland. - Ecological Studies, 150: 57-65.

HANSEN E.S., 2008: A contribution to the lichen flora of Kuhn $\varnothing$ and Blåbærdalen, easternmost Th. Thomsen Land, North East Greenland. Cryptogamie, Mycologie 29(4): 397-411.

HANSEN E.S., 2009a: A contribution to the lichen flora of Hold with Hope and Hudson Land, North East Greenland. - Cryptogamie, Mycologie, 30(3): 269-278.
HANSEN E.S., 2009b: Lichens from Johannes V. Jensen Land, N Greenland, the northernmost arctic land area. - Willdenowia, 39: 179-186.

HANSEN E.S., 2012: A contribution to the lichen flora of North East Greenland. - Botanica Lithuanica, 18(2): 109-116.

Hansen E.S., Aнti T., 2011: A contribution to the lichen genus Cladonia in Greenland and new records from other northern regions. - Graphis Scripta, 23: 56-64.

JAKOBSEN B.H., 1992: Zackenbergområdets naturgeografi. - Naturens Verden, 8: 306-313.

Koch L., Haller J., 1971: Geological map of East Greenland $72^{\circ}-76^{\circ} \mathrm{N}$. Lat. (1:250.000). - Meddelelser om Grønland, 183: 1-26 and 13 map sheets.

LyNGE B., 1940: Lichens from North East Greenland. II. Microlichens. - Skrifter om Svalbard og Ishavet, 81: 1-143.

Lynge B., Scholander P.F., 1932: Lichens from North East Greenland, 41: 1-116.

Meltofte H., Christensen T.R., Elberling B., ForchHAMMER M., RASCH M.C. (eds), 2008: High-Arctic Ecosystem Dynamics in a Changing Climate. Ten year of monitoring and research at Zackenberg Research Station, Northeast Greenland. - Advances in Ecological Research, 40. - Amsterdam.

Nordin A., Moberg R., Tønsberg T., Vitikainen O., Dalsätt Å., Myrdal M., Snitting D., Ekman S., 2011: Santesson's Checklist of Lichen-forming and Lichenicolous Fungi. - http://130.238.83.220/ santesson/home.php [Accessed 9 December 2016].

SAAG L., SAAG A., Randlane T., 2007: Survey of $L e-$ praria and Leprocaulon in Greenland. - Mycotaxon, 102: 57-90.

Thomson J.W., 1997: American Arctic lichens. II. The microlichens. - Wisconsin.

\section{PAPILDOMI DUOMENYS APIE PIETRYČIUU GRENLANDIJOS KERPIŲ FLORĄ V. ZAKENBERGO IR KLAVERINGO Ø VIETOVĖS}

\section{Eric Steen Hansen}

\section{Santrauka}

Straipsnyje pateikiamas 202 kerpių taksonų sąrašas iš šiaurès rytinès Grenlandijos, Zakenbergo ir Klaveringo Ø vietovių. Trys kerpių rūšys: Ochrolechia androgyna, Psora globifera ir Rhizocarpon renneri rastos pirmą kartą rytinejje Grenlandijoje. Šešiolika kerpių rūšiu yra naujos šiaurès rytinei Grenlandijai: Aspicilia aqua- tica, Candelariella dispersa, Chaenotheca furfuracea, Circinaria caesiocinerea, Cladonia libifera, Lecanora cenisia, L. chloroleprosa, L. leptacina, Lichenomphalia alpina, Miriquidica atrofulva, M. nigroleprosa, Ochrolechia alaskana, Peltigera castanea, P. extenuata, Phylliscum demangeonii ir Pyrenopsis furfurea. 